\title{
MATHEMATICAL ANALYSIS OF THE OPTIMIZING ACQUISITION AND RETENTION OVER TIME PROBLEM*
}

\author{
Adi Ditkowski ${ }^{1}$
}

\begin{abstract}
While making informed decisions regarding investments in customer retention and acquisition becomes a pressing managerial issue, formal models and analysis, which may provide insight into this topic, are still scarce. In this study we examine two dynamic models for optimal acquisition and retention models of a monopoly, the total cost and the cost per customer models. These models are analytically analyzed using classical, direct, methods and asymptotic expansions (for the total cost model). In order to numerically simulated the models, an innovative numerical method was developed for solving ODE systems with initial/final value problems.
\end{abstract}

Mathematics Subject Classification. 34B15, 34B60, 34B93, 34C11, 34E05, 49N05, 65L10.

Received December 21, 2007. Revised July 3rd, 2008.

Published online November 14, 2008.

\section{INTRODUCTION}

The shift of many firms away from the product to the customer as a unit of analysis has drawn considerable attention to the use of customer equity as a central tool for firm's market analysis [13]. Using the customer equity approach, customers are viewed as assets that create long-term value for the firm. Managers are thus encouraged to optimize the investments in these assets over time in order to maximize the long-term net cash flow from their customers [21].

An important decision that the firm has to make in this respect concerns the magnitude and timing of its investments in customer acquisition and retention. The growing availability of customer data, coupled with increasing awareness regarding the long-term impact of successful acquisition and retention strategies, make these decisions a pressing managerial issue. For example, Morgan Stanley, aiming at shifting from a productcentric to a client-centric organization, has made it a goal "to make customer acquisition and retention a more analytical and measurable process" [16].

Such examples demonstrate the need for tools as well as intuitive processes that will guide managers when making their investments in customer acquisition and retention. However, in spite of the importance of this subject, the academic literature in this area is still limited, as only recently have marketers begun to rigorously examine the relationship between customer acquisition and retention [20,23]. Current approaches to this problem

\footnotetext{
Keywords and phrases. ODE nonlinear boundary value problems; ODE applications; ODE growth, boundedness, comparison of solutions; ODE asymptotic expansions; optimal control; numerical methods ODE boundary value problems.

* This research was supported by the ISRAEL SCIENCE FOUNDATION (grant No. 1364/04), the UNITED STATES-ISRAEL BINATIONAL SCIENCE FOUNDATION (grant No. 2004099) and the Israel Institute for Business Research.

${ }^{1}$ School of Mathematical Sciences, Tel Aviv University, Tel Aviv 69978, Israel. adid@post.tau.ac.il
} 
are geared for helping a given company set its acquisition and retention budgets at a specific point in time such that the profit at this time period is maximized [3], rather than providing the managerial insights and tools for optimizing the acquisition and retention budgets such that the total profit over the lifetime of the company will be maximized.

While historically much attention has been devoted in the marketing literature to offensive actions aimed at acquiring new customers, since the mid-1980s, increasing attention has been paid to defensive strategies, or actions focusing on retaining existing customers [27]. In one of the first rigorous treatments of this issue, Fornell and Wernerfelt [9] studied various complaint handling programs that marketers use to affect retention, and concluded that in general, more attention should be paid to defensive strategies. Their theoretical findings were later followed by a series of papers by practitioners and academics alike that pointed to the need to focus on retention, given its effect on the long-term bottom line (e.g., see [27]). Given its effect on the valuation of customers, the retention rate has also been suggested as an important factor affecting the value of firms in general [11].

As the focus on retention spending has become an important managerial issue and the basis for many of the investments in CRM systems, recent doubts have been raised as to whether this focus has gone too far, coming at the expense of profits and the growth of the company through new customers $[1,19]$. Models and analysis of the acquisition/retention problem can potentially help in clarifying this point. However, while the long-run optimal balance of acquisition and retention budget allocation is recognized as one of the challenging tasks facing marketing resource allocation in general [12], there is scant formal analysis of these issues in the marketing literature.

\subsection{Customer equity in a growing market}

Customer lifetime value (CLV) has gained increasing interest in recent years as a basic tool to help firms determine the magnitude and the nature of investment in their customer relationships [4,14,19,21]. CLV is defined as: the discounted cash flow a firm expects to receive from an individual customer over some extended period $(t \rightarrow \infty)$. An example of a CLV model is, for example, if the retention rate is $r$, the average profit from a customer is $p$, and the discount rate is $\delta$, then the CLV over a long time horizon (as $t$ approaches infinity) is:

$$
\mathrm{CLV}=\sum_{t=0}^{\infty} \frac{p r^{t}}{(1+\delta)^{t+1}}=\frac{p}{(1+\delta-r)}
$$

This is a discrete model which assumes that all the parameters $p, \delta$ and $r$ remain constant in time.

This formulation may be slightly modified based on specific assumptions regarding the exact time that the cash flow is received during each period, and the time during a given period when a defection is assumed to have occurred [10].

Note that the above formula assumes that when customers leave the firm, they do not come back (or if they do, they are considered new customers). This "lost for good" assumption, which enables a relatively straightforward modelling of CLV, may be less robust in markets where consumers switch often among brands, such as with frequently purchased goods, and in such cases may result in the underestimation of the actual CLV. In such cases "migration models", which utilize a Markov chain analysis may be a better fit [21].

Equation (1.1) represents the lifetime value of a single customer. When maximizing their long-range profit, firms will be more interested in customer equity, or the sum of the lifetime value of all of the firm's customers $[21,25]$. While most applications have examined customer equity in the context of the value of the current customer base, see for example [3,4,21], suggest that customer equity should also include the value of future customers. Indeed, recent use of customer equity for firm valuation took into account the acquisition of new customers in a growing market [11].

The modelling of customer equity in a growing market is clearly important given the central role of new products in the sales of many firms. The importance of such cases is especially visible where customer equity models are used to calculate the value of firms, especially for service firms such as electronic commerce retailers 
for whom customer relations are recognized as major assets [10]. For example, it has been recently suggested that customer equity measures should play a much more central role in decisions regarding firms' mergers and acquisitions [22]. Therefore, since the firm's value depends on future cash flow from its customers, all customers should be included in the calculations, including anticipated new customers. To differentiate this approach from customer equity measures that are based on the current customer base, we label the customer equity in a growing market as dynamic customer equity (DCE).

\subsection{Dynamic customer equity (DCE)}

This basic formulation of the dynamic customer equity equation is similar in nature to the one presented in [11], therefore it is only briefly presented here. Historically, most formulations of the "lost for good" CLV analysis have been discrete. However, the derivation of a continuous analogy of the discrete case is essential for the formulation of a maximization problem.

Starting with the individual CLV, let $x(t)$ be the state variable that denotes the probability that a consumer remains an active customer of the firm at time $t$. This implies that

$$
\frac{\mathrm{d} x(t)}{\mathrm{d} t}=-(1-r) x(t), \quad x(0)=1 .
$$

For constant $r$ the solution of $(1.2)$ is

$$
x(t)=\mathrm{e}^{-(1-r) t} .
$$

The continues analog of (1.1) becomes

$$
\mathrm{CLV}=\int_{t=0}^{\infty} p x \mathrm{e}^{-\delta t} \mathrm{~d} t=\int_{t=0}^{\infty} p \mathrm{e}^{-(1-r) t} \mathrm{e}^{-\delta t} \mathrm{~d} t=\frac{p}{(1+\delta-r)}
$$

We thus assume that the lifetime value of a single customer is as shown in (1.4). Since the firm acquires more customers over time, the cumulative number of customers acquired by the firm grows with time (customers may of course leave after they are acquired, depending on the retention rate). Since our calculations start at time zero, the sum of the lifetime value of each group of customers acquired at time $t$ should be discounted to time zero. Let $a(t)$ be the cumulative number of acquired customers up to time $t$. If we assume a general growth function of $\mathrm{d} a / \mathrm{d} t=f(t)$ where $f(t)$ is a general continuous growth function, then the general form of the dynamic customer equity is given by

$$
\mathrm{DCE}=\int_{t=0}^{\infty} \int_{s=t}^{\infty} p \mathrm{e}^{-(1+\delta-r)(s-t)} f(t) \mathrm{e}^{-\delta t} \mathrm{~d} s \mathrm{~d} t
$$

which is similar to the one derived in [11].

\subsection{Dynamic optimal acquisition and retention of a monopolist}

In a recent manuscript [8], dynamic optimal acquisition and retention of a monopolist models were derived. Under this dynamic setting, the firm can use its retention and acquisition budgets to control the growth and maintenance of its customer base. In the context of this model, retention spending will affect the retention rate $r$ that becomes $r(\hat{t})^{1}$, while acquisition spending affects the growth rate of the number of new customers, represented by $g(\hat{t})$. Under this formulation, a change in retention spending affects the retention rate of all customers - new and old. Note that $x(\hat{t})$, which was the fraction of active customers for a given cohort in (1.2) becomes in the dynamic model the percentage of customers out of the total customers acquired to date $\hat{a}(\hat{t})$

\footnotetext{
${ }^{1}$ It should be noted that for these models the parameters, variables and functions, which have units are marked with ^. The first step in the analysis will be to normalize these quantities. For the unit-less quantities, the ^ will be omitted. As for the time. The 'physical' (dimensional) time is denoted by $\hat{t}$. The discount rate $\delta$ has units of $1 / t$. The non-dimensional time will be denoted by $t$ and is defined as $t=\hat{t} \delta$.
} 
(i.e., of all cohorts) that are still active customers of the firm. Thus the firm can affect the rate of acquisition of new customers through its acquisition efforts, and the rate of retention through its retention efforts. Therefore the equations governing $x(\hat{t})$ and $g(\hat{t})$ are

$$
\begin{aligned}
& \frac{\mathrm{d} x}{\mathrm{~d} \hat{t}}=-\hat{\rho}(1-r) x \\
& \frac{\mathrm{d} \hat{a}}{\mathrm{~d} \hat{t}}=\hat{\gamma}(m-\hat{a}) g
\end{aligned}
$$

with the initial conditions

$$
x(0)=1, \quad \hat{a}(0)=0 .
$$

The constants $\hat{\rho}$ and $\hat{\gamma}$ are required for several reasons. Formally, the most important one, is to match the units in both sides of the equations ( $\hat{\rho}$ and $\hat{\gamma}$ have the units of $1 / t$ ).

Costs can take on two forms: either total costs, which are independent of the number of customers, or else cost per-customer. For acquisition costs, the difference between total and per-customer is a choice of convenience only. If we denote the total acquisition costs by $\hat{K}(g)$, and the acquisition cost per potential customer by $\hat{k}(g)$, then the relationship between the two is given by the following: $\hat{K}(g)=(\hat{m}-\hat{a}) \hat{k}(g)$, where $\hat{a}(t)$ is the cumulative number of acquired customers up to period $t$, and $\hat{m}$ is the market potential. Consistent with [3], we assume the cost functions to be monotonic and convex.

One should note that assuming a convex cost function is equivalent to assuming a concave effectiveness function. The concavity is the manifestation of the diminishing marginal utility of the effectiveness function. The model given in [3] is the concave effectiveness retention function $r(\hat{c})=\bar{r}\left(1-\mathrm{e}^{-\hat{\alpha} \hat{c}}\right)$, where $\hat{c}$ is the dollar cost. This function is equivalent to the convex cost function $\hat{c}(r)=(1 / \hat{\alpha}) \log (\bar{r} /(\bar{r}-r))$, where $r$ is the retention resulting from expenditures of $\hat{c}$.

In the retention costs case, the difference between total costs and per-customer cost is much more pronounced and case-dependent. Since the retention rate $r$ is bounded, by definition, between 0 and 1 , the question is whether it depends on the number of consumers, or on the market potential alone. For example, for many Web-based firms, retention and the resultant repeat purchasing depend on factors such as categorizing users by their technological sophistication, ensuring perceived security, empowering users, and creating trust and commitment [24], none of which depend on the number of users. In contrast, the retention efforts of many brick-and-mortar firms such as call centers do depend on the number of users. We thus separate our analysis into these types of costs. As we demonstrate, the results are inherently similar, though not all results could be replicated for both cases.

For the analysis, it is assumed that the cost functions $\hat{C}(r)$ and $\hat{K}(g)$ satisfy

$$
\hat{C}(0)=\hat{C}^{\prime}(0)=\hat{K}(0)=\hat{K}^{\prime}(0)=0
$$

and

$$
\hat{C}^{\prime}(s), \hat{K}^{\prime}(s)>0 \quad \forall s>0 ; \quad \hat{C}^{\prime \prime}(s), \hat{K}^{\prime \prime}(s)>0 \quad \forall s \geq 0 .
$$

It is also assumed that $\hat{C}, \hat{C}^{\prime}, \hat{C}^{\prime \prime}, \hat{K}, \hat{K}^{\prime}$ and $\hat{K}^{\prime \prime}$ are smooth and bounded, and similarly, the same assumptions hold for $\hat{c}$ and $\hat{k}$.

In this paper we analyze the two monopoly models, derived in [8]. In Section 2, the total cost model is derived and analyzed. The analysis was done using direct estimates and asymptotic methods. The results are compared to numerical simulations. In Section 3, the cost per-customer model is presented and analyzed. Conclusions are given is Section 4.

Though the equations in this paper are quite different than the ones in $[5,7]$, the analysis done in this work was highly influenced by these manuscripts. 


\section{The total Cost Model}

In this model the $\widehat{\mathrm{DCE}}$ is defined by

$$
\widehat{\mathrm{DCE}}=\int_{0}^{\hat{T}}[\hat{p} \hat{a} x-\hat{C}(r)-\hat{K}(g)] \mathrm{e}^{-\delta \hat{t}} \mathrm{~d} \hat{t},
$$

which is the integral over the lifetime of the firm, $\hat{T}$, of the discounted net profit (income, $\hat{p} \hat{a} x$, minus acquisition and retention expanses, $\hat{C}(r)+\hat{K}(g))$.

The goal is to maximize $\widehat{\mathrm{DCE}}$ with respect to $r(\hat{t})$ and $g(\hat{t})$, subject to the constraints $(1.6)$ and $(1.7)$ with the initial conditions (1.8). $r(\hat{t})$ and $g(\hat{t})$ are restricted such that

$$
0 \leq r(\hat{t}) \leq 1, \quad g(\hat{t}) \geq 0
$$

The functions $\hat{C}$ and $\hat{K}$ are assumed to satisfy (1.9), (1.10) and the smoothness conditions listed above.

We shall now present the following normalization

$$
a=\frac{\hat{a}}{\hat{m}}, \quad \hat{P}=\hat{p} \hat{m} \delta, \quad t=\delta \hat{t}
$$

and define

$$
E=\frac{\widehat{\mathrm{DCE}}}{\int_{0}^{\infty} \hat{p} \hat{m} \mathrm{e}^{-\delta \hat{t}} \mathrm{~d} \hat{t}}=\frac{\widehat{\mathrm{DCE}}}{\hat{P} / \delta}
$$

The new value $E$ can be viewed as an efficiency factor with respect to a 'perfect world' in which all the populations are customers of the firm, for all times and no expense is required to maintain them.

After the normalization (2.3)-(2.4), the optimization problem (2.1)-(2.2) turns into maximizing

$$
E=\int_{0}^{T}[a x-C(r)-K(g)] \mathrm{e}^{-t} \mathrm{~d} t
$$

with respect to $r(t)$ and $g(t)$, subject to

$$
\begin{aligned}
& \frac{\mathrm{d} x}{\mathrm{~d} t}=-\rho(1-r) x \\
& \frac{\mathrm{d} a}{\mathrm{~d} t}=\gamma(1-a) g
\end{aligned}
$$

where

$$
\rho=\frac{\hat{\rho}}{\delta}, \quad \gamma=\frac{\hat{\gamma}}{\delta}, \quad T=\delta \hat{T}, \quad C=\frac{\hat{C}}{\hat{P} / \delta}, \quad K=\frac{\hat{K}}{\hat{P} / \delta} .
$$

This is an isoperimetric problem and can be solved as follows, see for example $[26]^{2}$. Let:

$$
A=a+\epsilon \xi_{1}, \quad X=x+\epsilon \xi_{2}, \quad R=r+\epsilon \xi_{3}, \quad G=g+\epsilon \xi_{4},
$$

where $a, x, r$ and $g$ are the solutions of the optimization problem $(2.5)-(2.8), \epsilon$ is a small parameter and $\xi_{1}, \ldots, \xi_{4}$ are arbitrary functions, such that $\xi_{1}(0)=\xi_{2}(0)=0$.

We now define the function $\hat{E}$ as:

$$
\hat{E}=\int_{0}^{T}\left\{[A X-C(R)-K(G)] \mathrm{e}^{-t}+\mu_{1}\left[\frac{\mathrm{d} A}{\mathrm{~d} t}-\gamma(1-A) G\right]+\mu_{2}\left[\frac{\mathrm{d} X}{\mathrm{~d} t}+\rho(1-R) X\right]\right\} \mathrm{d} t .
$$

\footnotetext{
${ }^{2}$ This problem can also be transformed to the same ODE system using the optimal control method.
} 
At the optimum, $\max (\hat{E}), \epsilon=0$. Therefore, for any $\xi_{1}, \ldots, \xi_{4}$

$$
\frac{\mathrm{d} \hat{E}}{\mathrm{~d} \epsilon}=0
$$

By equating the coefficients of $\xi_{1}, \ldots, \xi_{4}$ in (2.10) to 0 one obtains

$$
\begin{array}{rr}
\frac{\mathrm{d} \mu_{1}}{\mathrm{~d} t}=x \mathrm{e}^{-t}+\gamma \mu_{1} g & \mu_{1}(T)=0 \\
\frac{\mathrm{d} \mu_{2}}{\mathrm{~d} t}=a \mathrm{e}^{-t}+\rho \mu_{2}(1-r) & \mu_{2}(T)=0 \\
C^{\prime}(r) \mathrm{e}^{-t}+\mu_{2} \rho x=0 & \\
K^{\prime}(g) \mathrm{e}^{-t}+\mu_{1} \gamma(1-a)=0 . &
\end{array}
$$

$\mu_{1}$ and $\mu_{2}$ can be eliminated from (2.11)-(2.14) by taking the derivative of (2.13) and (2.14) with respect to $t$ and substituting $\mathrm{d} \mu_{1} / \mathrm{d} t$ and $\mathrm{d} \mu_{2} / \mathrm{d} t$ into them using (2.11) and (2.12). We get the following set of equations:

$$
\begin{aligned}
\frac{\mathrm{d} a}{\mathrm{~d} t} & =\gamma(1-a) g \text { or } \frac{\mathrm{d}(1-a)}{\mathrm{d} t}=-\gamma(1-a) g \\
\frac{\mathrm{d} x}{\mathrm{~d} t} & =-\rho(1-r) x \\
\frac{\mathrm{d}}{\mathrm{d} t}\left(K^{\prime}(g) \mathrm{e}^{-t}\right) & =-\gamma(1-a) x \mathrm{e}^{-t} \\
\frac{\mathrm{d}}{\mathrm{d} t}\left(C^{\prime}(r) \mathrm{e}^{-t}\right) & =-\rho x a \mathrm{e}^{-t}
\end{aligned}
$$

with the initial-final conditions

$$
a(0)=0, \quad x(0)=1, \quad g(T)=0, \quad r(T)=0 .
$$

Remark 2.1. In this and in the next model, there is a trivial solution; $a \equiv r \equiv g \equiv 0$. In this case, due to restriction (2.2), $g$ is artificially forced to be zero. This solution implies that the profit $M$, or the efficiency $E$ is zero. In this case there is no point of opening the business.

\subsection{Analysis of the total cost model}

Proposition 2.1. $0 \leq x(t) \leq 1$, and $x$ is a monotonically non-increasing function.

Proof. Rewriting (2.16) as

$$
\frac{\mathrm{d}}{\mathrm{d} t}\left[x \mathrm{e}^{\rho \int_{0}^{t}(1-r(s)) \mathrm{d} s}\right]=0 .
$$

Integrating from 0 to $t$ and using the initial condition, $x(0)=1$, one gets

$$
x(t)=\mathrm{e}^{-\rho \int_{0}^{t}(1-r(s)) \mathrm{d} s} .
$$

Since $0 \leq r(s) \leq 1,0<x(t) \leq 1$ and $x$ is a monotonically non-increasing function.

Proposition 2.2. $0 \leq a(t) \leq 1$, and $a$ is a monotonically increasing function.

Proof. Rewriting (2.15) as

$$
\frac{\mathrm{d}}{\mathrm{d} t}\left[(1-a) \mathrm{e}^{\gamma \int_{0}^{t} g(s) \mathrm{d} s}\right]=0
$$


and integrating between 0 to $t$, we get

$$
(1-a(t))=\mathrm{e}^{-\gamma \int_{0}^{t} g(s) \mathrm{d} s}
$$

The result follows using the same argument as in Proposition 2.1.

Proposition 2.3. $g(t)$ is a monotonically decreasing function of $t$.

Proof. By integrating (2.17) from $t$ to $T$ and using that $g(T)=0$, thus, by $(1.9), K^{\prime}(g(T))=0$, one obtains

$$
K^{\prime}(g(t))=\gamma \mathrm{e}^{t} \int_{t}^{T}(1-a(s)) x(s) \mathrm{e}^{-s} \mathrm{~d} s .
$$

Then, by taking the derivative of equation (2.24) with respect to $t$, one obtains

$$
K^{\prime \prime}(g(t)) g_{t}(t)=\gamma \mathrm{e}^{t} \int_{t}^{T}(1-a(s)) x(s) \mathrm{e}^{-s} \mathrm{~d} s-\gamma(1-a(t)) x(t) .
$$

Since $x(t)$ and $(1-a(t))$ are monotonically decreasing functions

$$
\begin{aligned}
\gamma \mathrm{e}^{t} \int_{t}^{T}(1-a(s)) x(s) \mathrm{e}^{-s} \mathrm{~d} s & \leq \gamma \mathrm{e}^{t}(1-a(t)) x(t) \int_{t}^{T} \mathrm{e}^{-s} \mathrm{~d} s \\
& =\gamma(1-a(t)) x(t)\left(1-\mathrm{e}^{-(T-t)}\right) .
\end{aligned}
$$

Thus,

$$
K^{\prime \prime}(g(t)) g_{t}(t) \leq-\gamma(1-a(t)) x(t) \mathrm{e}^{-(T-t)}<0
$$

and since, by assumption (1.10), $K^{\prime \prime}(g(t))>0$ for all $g(t) \geq 0, g_{t}(t)<0$. Note that by $(2.2), g(t)$ is restricted to be nonnegative.

Proposition 2.4. As long as $0 \leq r \leq 1, r_{t}(0) \geq 0$ and $r_{t}(T)<0$.

Proof. As in the last proposition, by integrating (2.18) from $t$ to $T$

$$
C^{\prime}(r(t))=\rho \mathrm{e}^{t} \int_{t}^{T} a(s) x(s) \mathrm{e}^{-s} \mathrm{~d} s .
$$

Then, by taking the derivative of equation (2.28) with respect to $t$, one obtains

$$
C^{\prime \prime}(r(t)) r_{t}(t)=\rho \mathrm{e}^{t} \int_{t}^{T} a(s) x(s) \mathrm{e}^{-s} \mathrm{~d} s-\rho a(t) x(t) .
$$

At $t=0, a(t=0)=0$ and

At $t=T$,

$$
C^{\prime \prime}(r(0)) r_{t}(0)=\rho \int_{0}^{T} a(s) x(s) \mathrm{e}^{-s} \mathrm{~d} s \geq 0
$$

$$
C^{\prime \prime}(r(T)) r_{t}(T)=-\rho a(T) x(T)<0
$$

Note that, in principle, as $t$ approaches 0, the RHS of (2.28), may be larger than $C^{\prime}(1)$. In this case $r$ is truncated. This saturation phenomenon is called 'blitz'. 


\subsection{Estimates}

In this section we obtain finer estimates for the solutions of (2.15)-(2.18). Here, it is assumed that there is no 'blitz', i.e. the solution is smooth. It is also assumed that $T \gg 1$, thus, for most of the business life expectancy $\mathrm{e}^{-(T-t)} \ll 1$.

We shall now assume that

and

$$
k_{0} g \leq K^{\prime}(g) \leq k_{1} g, \quad 0<k_{0} \leq k_{1}
$$

$$
c_{0} r \leq C^{\prime}(r) \leq c_{1} r, \quad 0<c_{0} \leq c_{1}
$$

Recalling that $1 \geq(1-a)>0$, or $0 \leq a<1$, and from (2.21)

$$
\mathrm{e}^{-\rho t} \leq x \leq 1
$$

Then, substituting (2.32) into (2.24)

$$
\begin{aligned}
k_{0} g(t) \leq K^{\prime}(g(t)) & =\gamma \mathrm{e}^{t} \int_{t}^{T}(1-a(s)) x(s) \mathrm{e}^{-s} \mathrm{~d} s \\
& \leq \gamma \mathrm{e}^{t} \int_{t}^{T} \mathrm{e}^{-s} \mathrm{~d} s=\gamma\left(1-\mathrm{e}^{-(T-t)}\right) .
\end{aligned}
$$

Thus

Now, using (2.23) and (2.36)

$$
g(t) \leq \frac{\gamma}{k_{0}}\left(1-\mathrm{e}^{-(T-t)}\right)
$$

$$
\begin{aligned}
& (1-a(t))=\mathrm{e}^{-\gamma \int_{0}^{t} g(s) \mathrm{d} s} \geq \mathrm{e}^{-\gamma \int_{0}^{t} \frac{\gamma}{k_{0}}\left(1-\mathrm{e}^{-(T-s)}\right) \mathrm{d} s} \\
& =\mathrm{e}^{-\frac{\gamma^{2}}{k_{0}} t} \mathrm{e}^{\frac{\gamma^{2}}{k_{0}} \mathrm{e}^{-T}\left(\mathrm{e}^{t}-1\right)} \geq \mathrm{e}^{-\frac{\gamma^{2}}{k_{0}} t},
\end{aligned}
$$

therefore

$$
a \leq 1-\mathrm{e}^{-\frac{\gamma^{2}}{k_{0}} t}
$$

Using the fact that $a x<1$ and substituting (2.33) into (2.28) one gets

$$
\begin{aligned}
c_{0} r(t) \leq C^{\prime}(r(t)) & =\rho \mathrm{e}^{t} \int_{t}^{T} a(s) x(s) \mathrm{e}^{-s} \mathrm{~d} s \\
& \leq \rho \mathrm{e}^{t} \int_{t}^{T} \mathrm{e}^{-s} \mathrm{~d} s=\rho\left(1-\mathrm{e}^{-(T-t)}\right) .
\end{aligned}
$$

Thus

For $t \ll T$, using (2.38) one obtains

$$
r(t) \leq \min \left\{1, \frac{\rho}{c_{0}}\left(1-\mathrm{e}^{-(T-t)}\right)\right\} .
$$

$$
\begin{aligned}
c_{0} r(t) \leq C^{\prime}(r(t)) & \leq \rho \mathrm{e}^{t} \int_{t}^{T}\left[1-\mathrm{e}^{-\frac{\gamma^{2}}{k_{0}} s}\right] \mathrm{e}^{-s} \mathrm{~d} s \\
& =\rho\left[1-\mathrm{e}^{-(T-t)}+\frac{1}{1+\gamma}\left(-\mathrm{e}^{-\gamma t}+\mathrm{e}^{-\gamma T} \mathrm{e}^{-(T-t)}\right)\right] \\
& =\rho\left[1-\frac{1}{1+\gamma} \mathrm{e}^{-\gamma t}\right]+O\left(\mathrm{e}^{-(T-t)}\right) .
\end{aligned}
$$


Thus, for $t \ll T$

$$
r(t) \leq \min \left\{1, \frac{\rho}{c_{0}}\left(1-\frac{1}{1+\gamma} \mathrm{e}^{-\gamma t}\right)\right\}
$$

Since

$$
1 \geq(1-a(t)) \geq \mathrm{e}^{-\frac{\gamma^{2}}{k_{0}} t}
$$

and

$$
1 \geq x(t) \geq \mathrm{e}^{-\rho t}
$$

then, using (2.24), again

$$
\begin{aligned}
K^{\prime}(g(t)) & =\gamma \mathrm{e}^{t} \int_{t}^{T}(1-a(s)) x(s) \mathrm{e}^{-s} \mathrm{~d} s \\
& \geq \gamma \mathrm{e}^{t} \int_{t}^{T} \mathrm{e}^{-\frac{\gamma^{2}}{k_{0}} s} \mathrm{e}^{-\rho s} \mathrm{e}^{-s} \mathrm{~d} s \\
& =\gamma \frac{1}{\left(1+\rho+\frac{\gamma^{2}}{k_{0}}\right)} \mathrm{e}^{-\frac{\gamma^{2}}{k_{0}} t}\left(1-\mathrm{e}^{-(T-t)}\right) .
\end{aligned}
$$

Thus

$$
\begin{aligned}
g(t) & \geq \frac{\gamma}{k_{1}\left(1+\rho+\frac{\gamma^{2}}{k_{0}}\right)} \mathrm{e}^{-\frac{\gamma^{2}}{k_{0}} t}\left(1-\mathrm{e}^{-(T-t)}\right) \\
& =\frac{\gamma}{k_{1}\left(1+\rho+\frac{\gamma^{2}}{k_{0}}\right)} \mathrm{e}^{-\frac{\gamma^{2}}{k_{0}} t}\left[1+O\left(\mathrm{e}^{-(T-t)}\right)\right]
\end{aligned}
$$

\subsection{Asymptotic expansions}

For this model analytic approximation can be derived for the case of large $K$ and $C$. This situation is the case where the expenses for both gaining new customers and maintaining old ones are large with respect to the profit $p$, see (2.8).

In the case where $K$ and $C$ are small, as can be seen in Figure $3, r=1$ in most of the domain, $t \in[0, T]$ and there are sharp transients in $a$ and $g$ near $t=0$. This may suggest that a boundary layer is formed. The standard method of analysis in such case is using the match asymptotic expansions, see for example [2]. This approach assumes two limits, an inner solution, inside the boundary layer which corresponds to 'small' $t$, and an outer solution for 'large' $t$. These solutions should be matched in an intermediate area. When the method of match asymptotic expansions was applied here, these solutions couldn't be matched. This indicates that the boundary condition here has a more complex structure. This line of research was thus deferred to later study.

There are other possibilities for expansion, however, these cases do not correspond to any real-life scenario.

Formally, we substitute,

$$
K=\frac{\tilde{K}}{\varepsilon}, \quad C=\frac{\tilde{C}}{\varepsilon},
$$

where $\tilde{K}$ and $\tilde{C}$ are $O(1)$ and $1 / \varepsilon$ is a 'typical' value for $K$ and $C$. For simplicity it is assumed that they are of the same order. 
We shall assume the following formal expansion:

$$
\begin{aligned}
a(t)=a_{0}(t)+\varepsilon a_{1}(t)+\varepsilon^{2} a_{2}(t)+\ldots, & a_{j}(0)=0 ; & j=0,1, \ldots \\
x(t)=x_{0}(t)+\varepsilon x_{1}(t)+\varepsilon^{2} x_{2}(t)+\ldots, & x_{0}(0)=1 ; x_{j}(0)=0 ; & j=1,2, \ldots \\
r(t)=r_{0}(t)+\varepsilon r_{1}(t)+\varepsilon^{2} r_{2}(t)+\ldots, & r_{j}(T)=0 ; & j=0,1, \ldots \\
g(t)=g_{0}(t)+\varepsilon g_{1}(t)+\varepsilon^{2} g_{2}(t)+\ldots, & g_{j}(T)=0 ; & j=0,1, \ldots
\end{aligned}
$$

and, using Taylor expansion,

$$
\begin{aligned}
& \tilde{K}^{\prime}(g)=\tilde{K}^{\prime}\left(g_{0}\right)+\varepsilon \tilde{K}^{\prime \prime}\left(g_{0}\right) g_{1}+\varepsilon^{2}\left[\tilde{K}^{\prime \prime}\left(g_{0}\right) g_{2}+\frac{\tilde{K}^{\prime \prime \prime}\left(g_{0}\right)}{2} g_{1}^{2}\right]+O\left(\varepsilon^{3}\right), \\
& \tilde{C}^{\prime}(r)=\tilde{C}^{\prime}\left(r_{0}\right)+\varepsilon \tilde{C}^{\prime \prime}\left(r_{0}\right) r_{1}+\varepsilon^{2}\left[\tilde{C}^{\prime \prime}\left(r_{0}\right) r_{2}+\frac{\tilde{C}^{\prime \prime \prime}\left(r_{0}\right)}{2} r_{1}^{2}\right]+O\left(\varepsilon^{3}\right) .
\end{aligned}
$$

Then

$$
\begin{aligned}
a= & \varepsilon a_{1}+O\left(\varepsilon^{2}\right)=\frac{\gamma^{2}}{\tilde{K}^{\prime \prime}(0)(\rho+1)}\left(\frac{1-\mathrm{e}^{-\rho t}}{\rho}-\mathrm{e}^{-\rho T} \mathrm{e}^{-(T-t)}\left(1-\mathrm{e}^{-t}\right)\right) \varepsilon+O\left(\varepsilon^{2}\right) \\
x= & x_{0}+O\left(\varepsilon^{2}\right)=\mathrm{e}^{-\rho t}+O\left(\varepsilon^{2}\right) \\
g= & \varepsilon g_{1}+O\left(\varepsilon^{2}\right)=\frac{\gamma}{\tilde{K}^{\prime \prime}(0)(\rho+1)} \mathrm{e}^{-\rho t}\left(1-\mathrm{e}^{-(\rho+1)(T-t)}\right) \varepsilon+O\left(\varepsilon^{2}\right) \\
r= & \varepsilon^{2} r_{2}+O\left(\varepsilon^{3}\right)=\frac{\gamma^{2}}{\tilde{C}^{\prime \prime}(0) \tilde{K}^{\prime \prime}(0)(\rho+1)^{2}(2 \rho+1)}\left[\mathrm{e}^{-t \rho}(2 \rho+1)-\mathrm{e}^{-2 t \rho}(\rho+1)\right. \\
& -\mathrm{e}^{t-T-(t+T) \rho}(2 \rho+1)(\rho+1)+2 \mathrm{e}^{t-T-2 T \rho}(\rho+1)^{2}-\mathrm{e}^{t-T(\rho+1)}(2 \rho+1) \\
& \left.+\mathrm{e}^{-T-(t+T) \rho} \rho(2 \rho+1)-\mathrm{e}^{t-2 T(\rho+1)} \rho(2 \rho+1)\right] \varepsilon^{2}+O\left(\varepsilon^{3}\right) .
\end{aligned}
$$

Note that for $T \ll 1$ and for $(T-t) \ll 1$, i.e. for long lasting business, far enough from its closing, (2.50) gets the following form:

$$
\begin{aligned}
a & =\frac{\gamma^{2}}{\tilde{K}^{\prime \prime}(0)(\rho+1)} \frac{1-\mathrm{e}^{-\rho t}}{\rho} \varepsilon+O\left(\varepsilon^{2}\right) \\
x & =\mathrm{e}^{-\gamma t}+O\left(\varepsilon^{2}\right) \\
g & =\frac{\gamma}{\tilde{K}^{\prime \prime}(0)(\rho+1)} \mathrm{e}^{-\rho t} \varepsilon+O\left(\varepsilon^{2}\right) \\
r & =\frac{\gamma^{2}}{\tilde{C}^{\prime \prime}(0) \tilde{K}^{\prime \prime}(0)(\rho+1)^{2}(2 \rho+1)}\left(\mathrm{e}^{-t \rho}(2 \rho+1)-\mathrm{e}^{-2 t \rho}(\rho+1)\right) \varepsilon^{2}+O\left(\varepsilon^{3}\right) .
\end{aligned}
$$

The expressions for $a_{2}, x_{2}$ and $g_{2}$ were omitted for clarity. 
From (2.50), it can be seen that $x(t)$ is exponentially decaying in $t$, and it is the only quantity that is of order 1. $a(t)$ is exponentially converging to $\frac{\gamma^{2}}{\tilde{K}^{\prime \prime}(0) \rho(\rho+1)} \varepsilon$ from below. $g(t)$ is exponentially decaying and is maximal value, at $t=0$ is $\frac{\gamma}{\bar{K}^{\prime \prime}(0)(\rho+1)} \varepsilon$ and $r(t)$ increases from $\rho \varepsilon^{2}$ to its maximal value at $t_{\max }=\frac{1}{\rho} \log \left(\frac{2(\rho+1)}{2 \rho+1}\right) \approx \frac{1}{\rho}$ and then decaying exponentially.

This approximation satisfies Propositions 2.1-2.4.

It should be emphasized that under the assumption of this asymptotic expansion, since $r(t)=O\left(\varepsilon^{2}\right)$ 'blitz' cannot occur. Furthermore, since, under these conditions, $r(t)=O\left(\varepsilon^{2}\right)$ and $g(t)=O(\varepsilon)$, it is highly important to gain new customers, and almost useless to preserve the existing ones.

\subsection{Numerical simulations}

In this section numerical solution of (2.15)-(2.18) are presented. The difficulty in simulating this problem is that integrating the variables $x$ and $a$ from the left boundary, $t=0$, to the right one, $t=T$, cannot be simply done, since $g$ and $r$ are not known up front. Similarly integrating $g$ and $r$ from $t=T$ to $t=0$ cannot be done because $a$ and $x$ are unknown. This problem suggest the use of relaxation techniques. In this methods, one starts with an initial 'guess' for the variables and uses it for solving the equations, after which the guess is updated. Several relaxation techniques are listed in the literature, see for example [18]. These methods work well when the solution is smooth, i.e. when there is no 'blitz'. A relaxation method that is not sensitive to the smoothness of the solution was suggested in [6]. The idea behind this method is to embed the original ODE system in a hyperbolic system, i.e.

$$
\begin{aligned}
\frac{\partial a}{\partial s} & =-\frac{\partial a}{\partial t}-\gamma(1-a) g \\
\frac{\partial x}{\partial s} & =-\frac{\partial x}{\partial t}-\rho(1-r) x \\
\frac{\partial}{\partial s}\left(K^{\prime}(g)\right) & =\frac{\partial}{\partial t}\left(K^{\prime}(g)\right)-\left(K^{\prime}(g)-\gamma(1-a) x\right) \\
\frac{\partial}{\partial s}\left(C^{\prime}(r)\right) & =\frac{\partial}{\partial t}\left(C^{\prime}(r)\right)-\left(C^{\prime}(r)-\rho x a\right) .
\end{aligned}
$$

This is a semi-linear hyperbolic system, in which $t \in[0, T]$ serves as the space variable and $s$ as the time. A steady state solution, $\partial / \partial s=0$, of this system is a solution of the original ODE system (2.15)-(2.18). The dynamics governed by this system is that the boundary conditions (2.19) are evolved along the characteristics $s-t=$ const. for $a$ and $x$, and $s+t=$ const. for $C^{\prime}$ and $K^{\prime}$. Though the solution for this system is not guaranteed to converge to a steady state (examples in which there is no convergence were constructed), the dynamics governed by this system is independent of the solution smoothness, or continuity. In practice, equations (2.52)-(2.55) are solved using any of the standard methods for solving hyperbolic systems, see for example [17]. For it's simplicity, the upwind scheme was used for the computations in this paper. $\Delta s$ were chosen to be equal to $\Delta t$. The basic spatial spacing, $\Delta t$ was $T / 500$, and for convergence study, it was refined to $T / 5000$. The scheme were run until it reached 'steady-state', in the sense that for more than 100 iterations, the difference between successive solution was less than $10^{-15}$. For more details, see [6].

In the examples given here, the cost functions $K(g)=K_{0} g^{2} / 2$ and $C(r)=C_{0} r^{2} / 2$ were chosen. These functions are commonly used in economics and marketing, see for example [15]. We had also chosen $\gamma=\rho=1$ and $T=50$.

As can be seen from Figure 1 , when $K_{0}$ and $C_{0}$ are fairly large $\left(K_{0}=C_{0}=100\right)$, there is a very good agreement between the asymptotic approximation and the numerical one. For more modest values of $K_{0}$ and $C_{0}\left(K_{0}=C_{0}=10\right)$, see Figure 2, the approximation is much worse. However, there is a reasonable error in the approximation of $x, g$ and $r$ over most of the domain $[0, T]$, and the asymptotic approximation to $a$ presents the same qualitative behavior as the numerical one. For small values of $K_{0}$ and $C_{0}$ (Fig. 3 ), where $\left(K_{0}=C_{0}=0.5\right)$, 

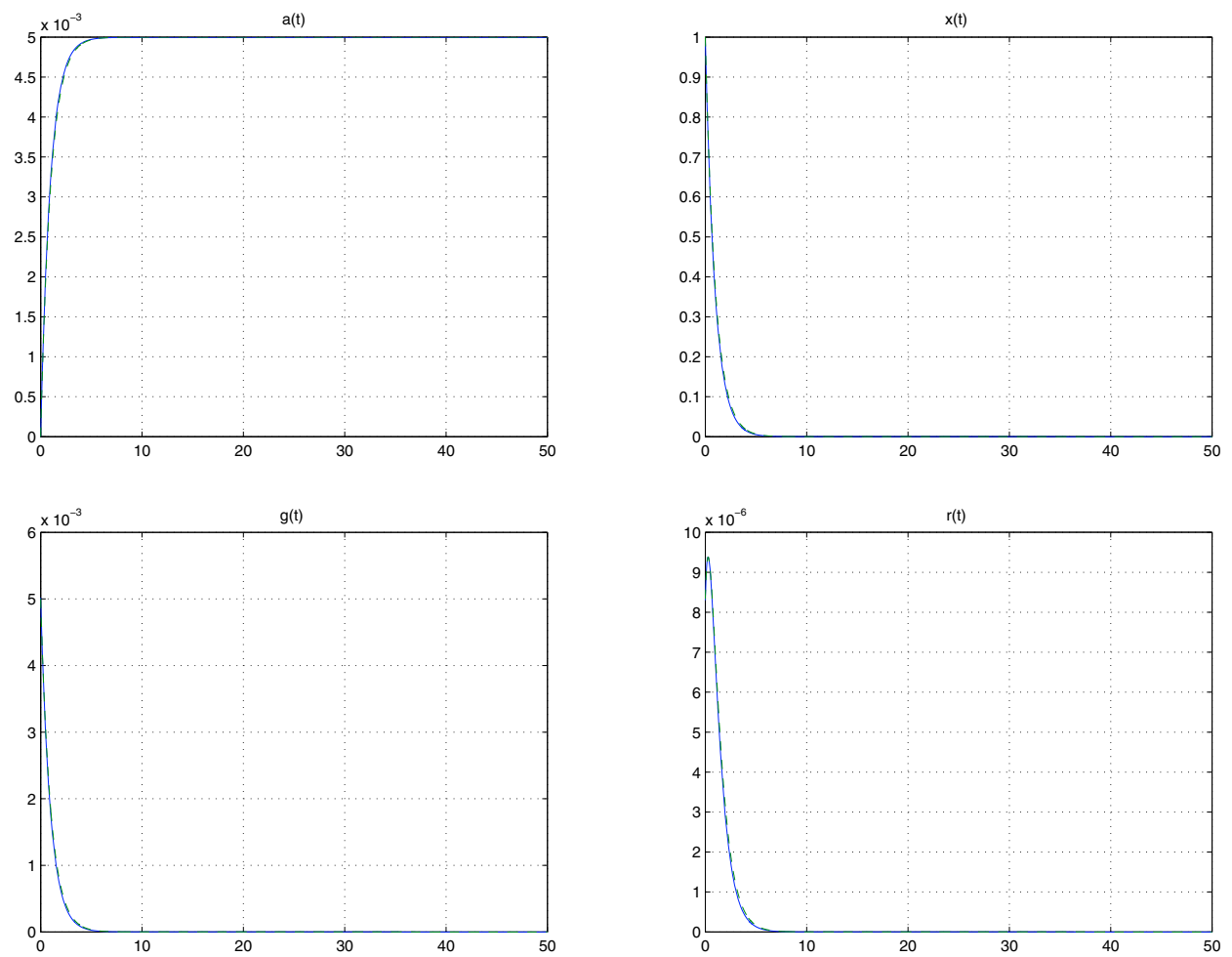

FIgure 1. Total cost model: numerical solutions in solid, asymptotic solutions in dashed. $\gamma=\rho=1, C_{0}=K_{0}=100$ and $T=50$. Top left, $a(t)$, top right, $x(t)$, bottom left, $g(t)$ and bottom right, $r(t)$.

there is a 'blitz' in $r$ for most of the domain and, as expected, there is no resemblances between the asymptotic and the numerical approximations.

The numerical results also agree with the analytic analysis and estimates. For large values of $K_{0}$ and $C_{0}, r$ reaches its maximal value very close to $t=0$, so Proposition 2.4 is satisfied.

It should also be noted that, using these parameters for $\gamma, \rho$ and $T$, the transition between no 'blitz' and 'blitz' for most of the time is very fast.

\section{The COST PeR CUSTOMer MOdeL}

Here the goal is to maximize

$$
\widehat{\mathrm{DCE}}=\int_{0}^{\hat{T}}[\hat{a} x(\hat{p}-\hat{c}(r))-(m-\hat{a}) \hat{k}(g)] \mathrm{e}^{-\delta \hat{t}} \mathrm{~d} \hat{t}
$$

with respect to $r(\hat{t})$ and $g(\hat{t})$, subject to the constraints, (1.6) and (1.7), the initial conditions (1.8), and the restrictions on $r(\hat{t})$ and $g(\hat{t}),(2.2)$. Note that here $\hat{c}$ and $\hat{k}$ are used instead of $\hat{C}$ and $\hat{K}$ in the previous model. However they have the same properties, (1.9), (1.10), and the same smoothness requirements as $\hat{C}$ and $\hat{K}$ from the previous model. 

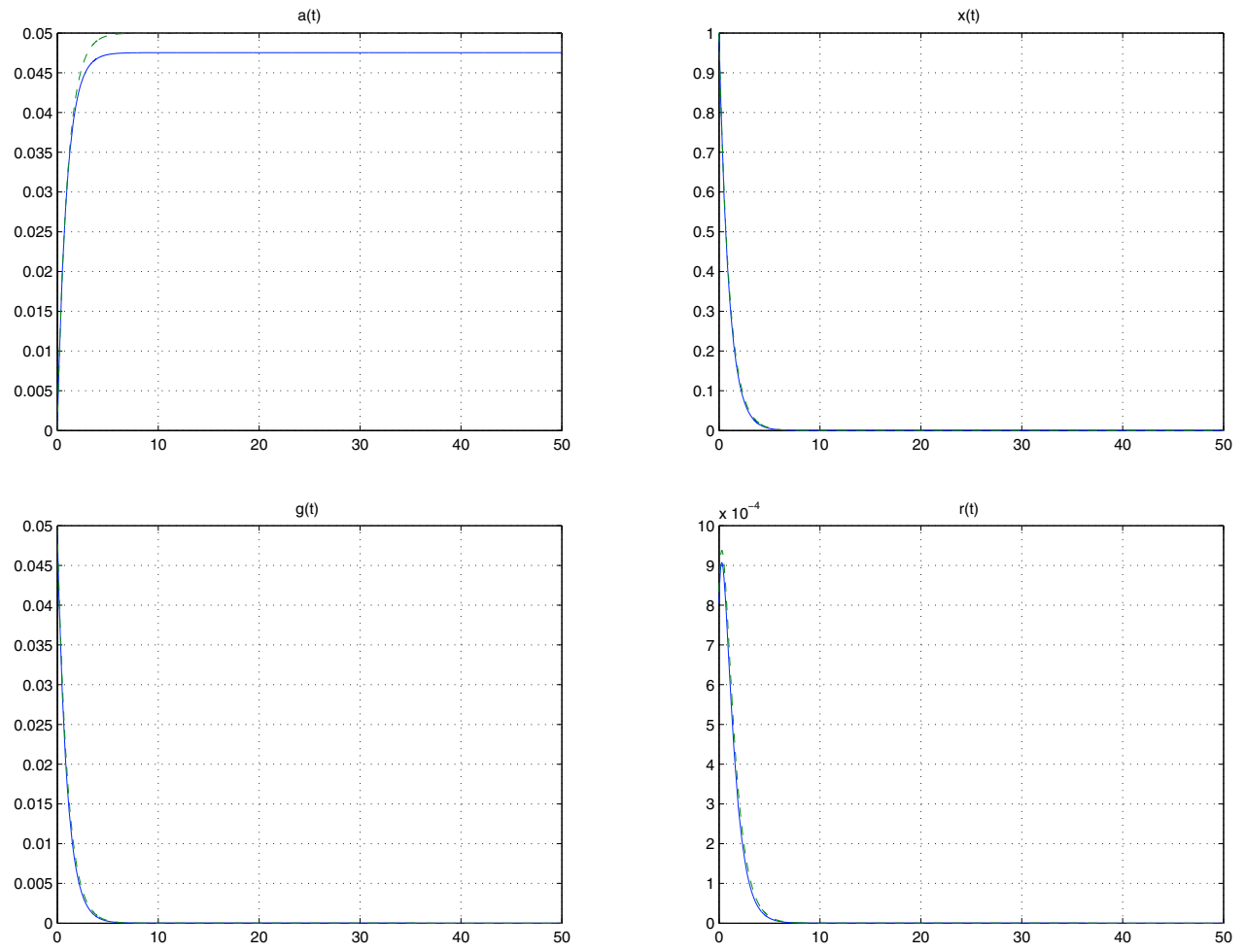

Figure 2. Total cost model: numerical solutions in solid, asymptotic solutions in dashed. $\gamma=\rho=1, C_{0}=K_{0}=10$ and $T=50$. Top left, $a(t)$, top right, $x(t)$, bottom left, $g(t)$ and bottom right, $r(t)$.

We use the same normalization, (2.3), (2.4) for $a, \hat{P}, t$ and $E$. However we replace the normalization for $\hat{C}$ and $\hat{K}((2.8))$ by:

$$
c=\frac{\hat{c} \hat{m}}{\hat{P} / \delta}, \quad k=\frac{\hat{k} \hat{m}}{\hat{P} / \delta}
$$

By solving a similar isoperimetric problem, and eliminating $\mu_{1}$ and $\mu_{2}$, as before, one obtains the following ODE system:

$$
\begin{aligned}
\frac{\mathrm{d} a}{\mathrm{~d} t} & =\gamma(1-a) g \text { or } \frac{\mathrm{d}(1-a)}{\mathrm{d} t}=-\gamma(1-a) g \\
\frac{\mathrm{d} x}{\mathrm{~d} t} & =-\rho(1-r) x \\
\frac{\mathrm{d}}{\mathrm{d} t}\left[(1-a) k^{\prime}(g) \mathrm{e}^{-t}\right] & =-\gamma(1-a) \mathrm{e}^{-t}[x(1-c(r))+k(g)] \\
\frac{\mathrm{d}}{\mathrm{d} t}\left(x a c^{\prime}(r) \mathrm{e}^{-t}\right) & =-\rho x a(1-c(r)) \mathrm{e}^{-t},
\end{aligned}
$$

with the initial-final conditions

$$
a(0)=0, \quad x(0)=1, \quad g(T)=0, \quad r(T)=0 .
$$



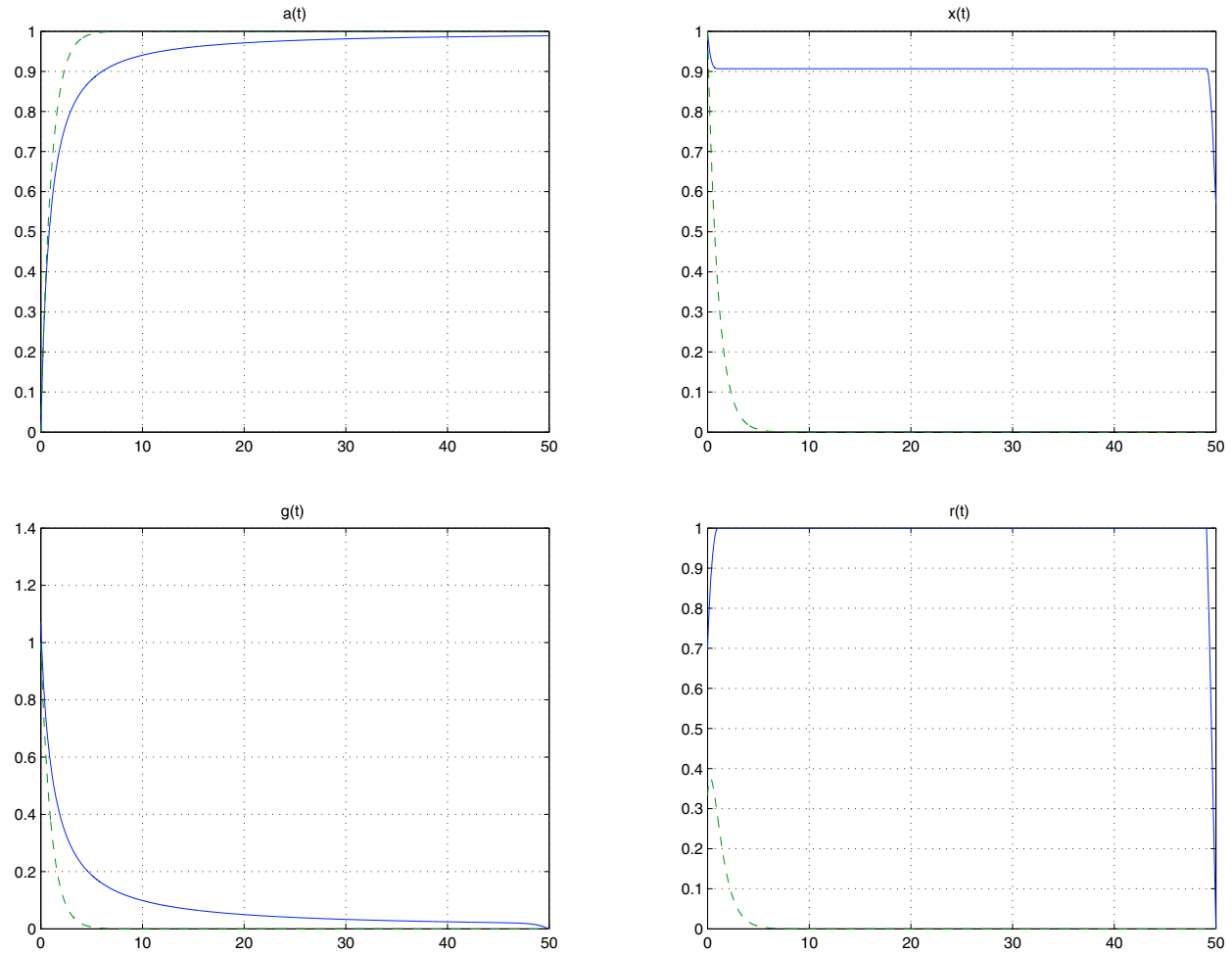

FIgure 3. Total cost model: numerical solutions in solid, asymptotic solutions in dashed. $\gamma=\rho=1, C_{0}=K_{0}=0.5$ and $T=50$. Top left, $a(t)$, top right, $x(t)$, bottom left, $g(t)$ and bottom right, $r(t)$.

As in the previous model, equations (3.5) and (3.6) can be integrated from $t$ to $T$ to get

$$
k^{\prime}(g(t))=\frac{\gamma \mathrm{e}^{t}}{1-a(t)} \int_{t}^{T}(1-a(s)) \mathrm{e}^{-s}[x(s)(1-c(r(s)))+k(g(s))] \mathrm{d} s
$$

and

$$
c^{\prime}(r(t))=\frac{\rho \mathrm{e}^{t}}{x(t) a(t)} \int_{t}^{T} x(s) a(s)(1-c(r(s))) \mathrm{e}^{-s} \mathrm{~d} s .
$$

\subsection{Analysis of the cost per customer model}

Proposition 3.1. $0 \leq x(t) \leq 1$, and $x$ is a monotonically non-increasing function.

Proposition 3.2. $1 \leq a(t) \leq 0$, and $a$ is a monotonically increasing function.

Proof. As they are the same, the proofs of Propositions 3.1 and 3.2 is identical to Propositions 2.1 and 2.2 in the previous section. Equations (2.21) and (2.23) also hold for this model.

Proposition 3.3. $c(r) \leq c^{\prime}(r)$.

Proof. By (1.10), $c^{\prime}(r)$ is a monotonically increasing function of $r$. Then

$$
c(r)=\int_{0}^{r} c^{\prime}(\tilde{r}) \mathrm{d} \tilde{r}=c^{\prime}\left(r^{*}\right) \int_{0}^{r} \mathrm{~d} \tilde{r} \leq c^{\prime}(r) r
$$


where $0 \leq r^{*}<r$. Since, by restriction (2.21), $0 \leq r^{*}<r \leq 1$,

$$
c(r) \leq c^{\prime}(r)
$$

Proposition 3.4. At $t=T, r_{t}$ and $g_{t}$ are negative.

Proof. From (3.6)

$$
\left(x a \mathrm{e}^{-t}\right) \frac{\mathrm{d}}{\mathrm{d} t}\left[c^{\prime}(r)\right]+\frac{\mathrm{d}}{\mathrm{d} t}\left(x a \mathrm{e}^{-t}\right) c^{\prime}(r)=-\rho x a(1-c(r)) \mathrm{e}^{-t} .
$$

Since at $t=T, r(T)=c^{\prime}(r(T))=0$

$$
\left(x(T) a(T) \mathrm{e}^{-T}\right) c^{\prime \prime}(0) r_{t}(T)=-\rho x(T) a(T) \mathrm{e}^{-T}
$$

and

Similarly, from (3.5)

$$
c^{\prime \prime}(0) r_{t}(T)=-\rho<0
$$

$$
(1-a) \mathrm{e}^{-t} \frac{\mathrm{d}}{\mathrm{d} t}\left[k^{\prime}(g)\right]+\frac{\mathrm{d}}{\mathrm{d} t}\left[(1-a) \mathrm{e}^{-t}\right] k^{\prime}(g)=-\gamma(1-a) \mathrm{e}^{-t}[x(1-c(r))+k(g)] .
$$

Also here, at $t=T, g(T)=k^{\prime}(r(T))=0$ and

$$
k^{\prime \prime}(0) g_{t}(T)=-\gamma x<0 .
$$

Proposition 3.5. There is always a 'blitz', i.e. there exists $0<t_{b}$ such that $r\left(t<t_{b}\right)=1$.

Proof. From Proposition 3.3, for all $0 \leq r \leq 1, c^{\prime}(r) \geq c(r)$. Then, from (3.5)

$$
\frac{\mathrm{d}}{\mathrm{d} t}\left(x a c^{\prime}(r) \mathrm{e}^{-t}\right)=\rho x a c(r) \mathrm{e}^{-t}-\rho x a \mathrm{e}^{-t} \leq \rho x a c^{\prime}(r) \mathrm{e}^{-t}-\rho x a \mathrm{e}^{-t} .
$$

Let

then (3.5) can be written as

$$
R \equiv x a c^{\prime}(r) \mathrm{e}^{-t}
$$

or

$$
R_{t}-\rho R \leq-\rho x a \mathrm{e}^{-t},
$$

By integrating (3.18) from $t$ to $T$ one obtains that

$$
\frac{\mathrm{d}}{\mathrm{d} t}\left(R \mathrm{e}^{-\rho t}\right) \leq-\rho x a \mathrm{e}^{-t} .
$$

$$
R \geq \rho \mathrm{e}^{\rho t} \int_{t}^{T} x(s) a(s) \mathrm{e}^{-(1+\rho) s} \mathrm{~d} s,
$$

or

$$
c^{\prime}(r) \geq \frac{\rho}{x(t) a(t)} \mathrm{e}^{(1+\rho) t} \int_{t}^{T} x(s) a(s) \mathrm{e}^{-(1+\rho) s} \mathrm{~d} s .
$$

Since the integral is positive and $x(0)=1, a(0)=0$,

$$
\lim _{t \rightarrow 0}\left[\frac{\rho}{x(t) a(t)} \mathrm{e}^{(1+\rho) t} \int_{t}^{T} x(s) a(s) \mathrm{e}^{-(1+\rho) s} \mathrm{~d} s\right]=\infty .
$$

The existence of 'blitz' now follows from (1.10). 
Proposition 3.6. $c^{\prime}(r) \geq \frac{\rho}{1+2 \rho}\left[1-\mathrm{e}^{-(1+2 \rho)(T-t)}\right]$.

Proof. By integrating (3.4) from $t$ to $s$ one obtains

$$
x(s)=x(t) \mathrm{e}^{-\rho \int_{t}^{s}(1-r(\sigma)) \mathrm{d} \sigma} .
$$

Thus for all $s \geq t$,

$$
\frac{x(s)}{x(t)}=\mathrm{e}^{-\rho \int_{t}^{s}(1-r(\sigma)) \mathrm{d} \sigma} \geq \mathrm{e}^{-\rho(s-t)} .
$$

From Proposition 3.2, it follows that for all $s \geq t$,

$$
\frac{a(s)}{a(t)} \geq 1
$$

By substituting the last two estimates into (3.20) one obtains

$$
\begin{aligned}
c^{\prime}(r) & \geq \frac{\rho}{x(t) a(t)} \mathrm{e}^{(1+\rho) t} \int_{t}^{T} x(s) a(s) \mathrm{e}^{-(1+\rho) s} \mathrm{~d} s \\
& \geq \rho \mathrm{e}^{(1+\rho) t} \int_{t}^{T} \mathrm{e}^{-\rho(s-t)} \mathrm{e}^{-(1+\rho) s} \mathrm{~d} s \\
& \geq \rho \mathrm{e}^{(1+2 \rho) t} \int_{t}^{T} \mathrm{e}^{-(1+2 \rho) s} \mathrm{~d} s \\
& =\frac{\rho}{1+2 \rho}\left[1+\mathrm{e}^{-(1+\rho)(T-t)}\right] .
\end{aligned}
$$

Therefore, for $T \gg 1$, there is a long 'maintenance', or a quasi steady-state, level, in which the optimal investment for retaining customers, $r$, is lager then $\rho /(1+2 \rho) \cdot r$ is dropping to zero only when the business is about to be closed.

Proposition 3.7. $g k^{\prime}(g) \geq k(g)$.

Proof. The proof is identical to the proof of Proposition 3.3.

Proposition 3.8. $g \leq k^{\prime-1}(\gamma) \cdot g_{t}(0)$ can be either positive or negative.

Proof. By expanding the LHS of (3.5) and using (3.3) one obtains

$$
\begin{aligned}
\frac{\mathrm{d}}{\mathrm{d} t} k^{\prime}(g)=k^{\prime \prime}(g) g_{t} & =(1+\gamma g) k^{\prime}(g)-\gamma[x(1-c(r))+k(g)] \\
& =k^{\prime}(g)+\gamma\left[g k^{\prime}(g)-k(g)\right]-\gamma x(1-c(r)) \\
& \geq k^{\prime}(g)-\gamma x(1-c(r)) .
\end{aligned}
$$

By integrating (3.25) from $t$ to $T$ one obtains

$$
k^{\prime}(g) \leq \gamma\left(1-\mathrm{e}^{-(T-t)}\right) \leq \gamma .
$$

Therefore, using (1.10)

$$
\text { At } t=0,
$$

$$
g \leq k^{\prime-1}(\gamma)
$$

$$
k^{\prime \prime}(g(0)) g_{t}(0) \geq k^{\prime}(g(0))-\gamma(1-C(1)) .
$$

The sign of the RHS of (3.28) is not a priori known. 

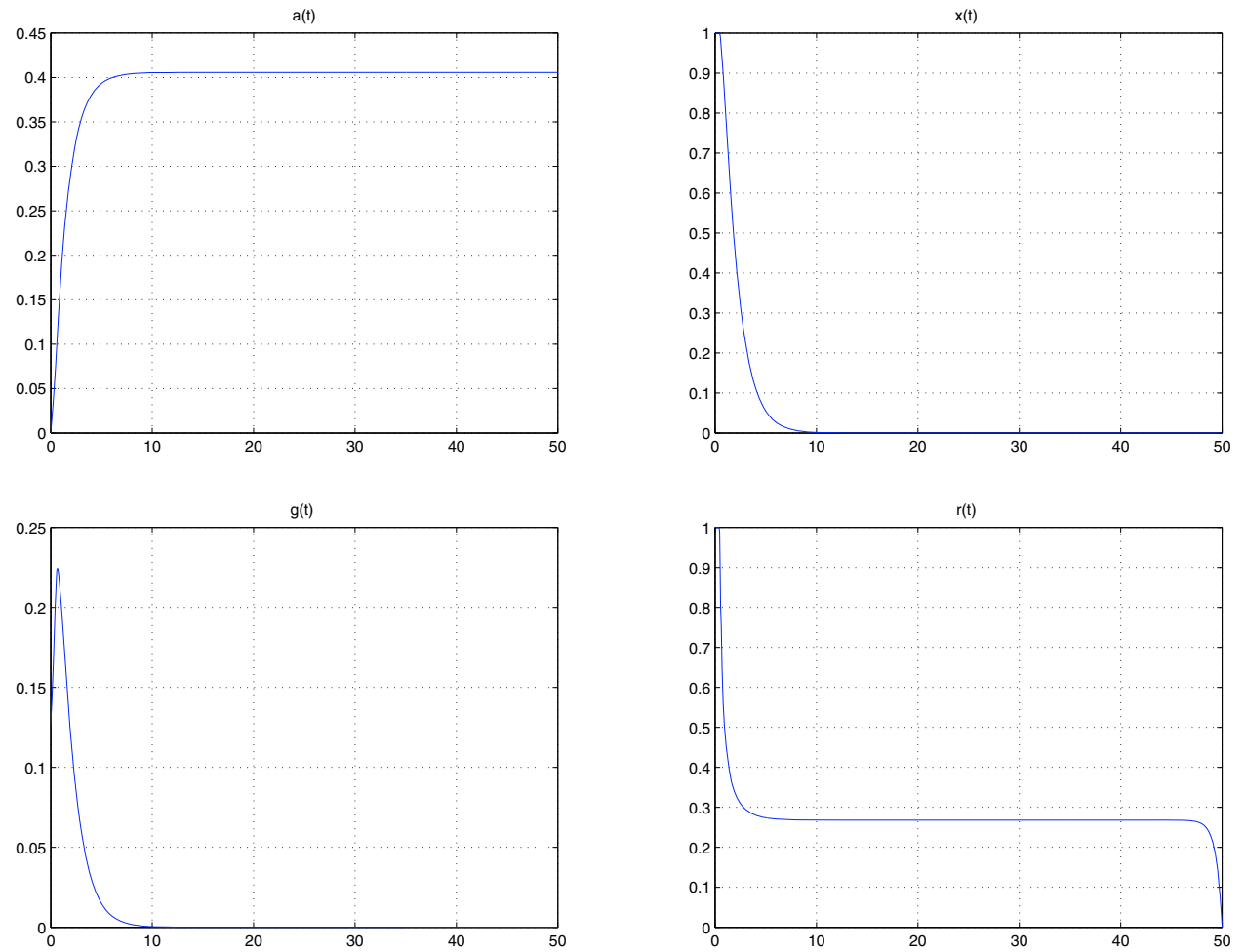

Figure 4. Cost per customer model: numerical solutions for $\gamma=\rho=1, k_{0}=c_{0}=2$ and $T=50$. Top left, $a(t)$, top right, $x(t)$, bottom left, $g(t)$ and bottom right, $r(t)$.

\subsection{Numerical simulations}

Unlike the total cost model in which for large $K$ and $C$ there was a scale separation, here, following Proposition 3.5, there is always a 'blitz', i.e. no matter how large the cost function $c$ is, $r$ will reach 1 for some time interval. Therefore the separation of scales does not happen here and it is almost as difficult to solve the approximated system as the original one. Therefore, no asymptotic analysis was done for this model.

As in the previous model, the cost model parameters were chosen as $k(g)=k_{0} g^{2} / 2$ and $c(r)=c_{0} r^{2} / 2$, $\gamma=\rho=1$ and $T=50$. The problem was solved using the same hyperbolic embedding as in the previous case.

Two types of solutions are presented here. Figure 4 shows the solution for 'large' $k_{0}$ and $c_{0}$. There is a short 'blitz' in $r$, followed by a long quasi stationary maintenance period until $r$ drops to 0 at the end of the business life. After the short 'blitz' $x$ is exponentially decaying to 0 . $a$ is exponentially converging to a constant and $g$ rises fast to a peak and then exponentially decaying.

The second type of solution, for 'small' $k_{0}$ and $c_{0}$ is presented in Figure 5. Here there is a 'blitz' for most of the time. $r, x$ and $g$ are 1 until close to $T$ and $a$ converges fast to 1 .

It should be noted that also in this case, in our experiments, the transient between very small 'blitz' interval and 'blitz' in most of the domain is very fast.

\section{Conclusions}

In [8] the first dynamic models for optimal accusation and retention were presented, namely the total cost and the cost per customer models. In this manuscript, these models are analyzed and numerically simulated. 

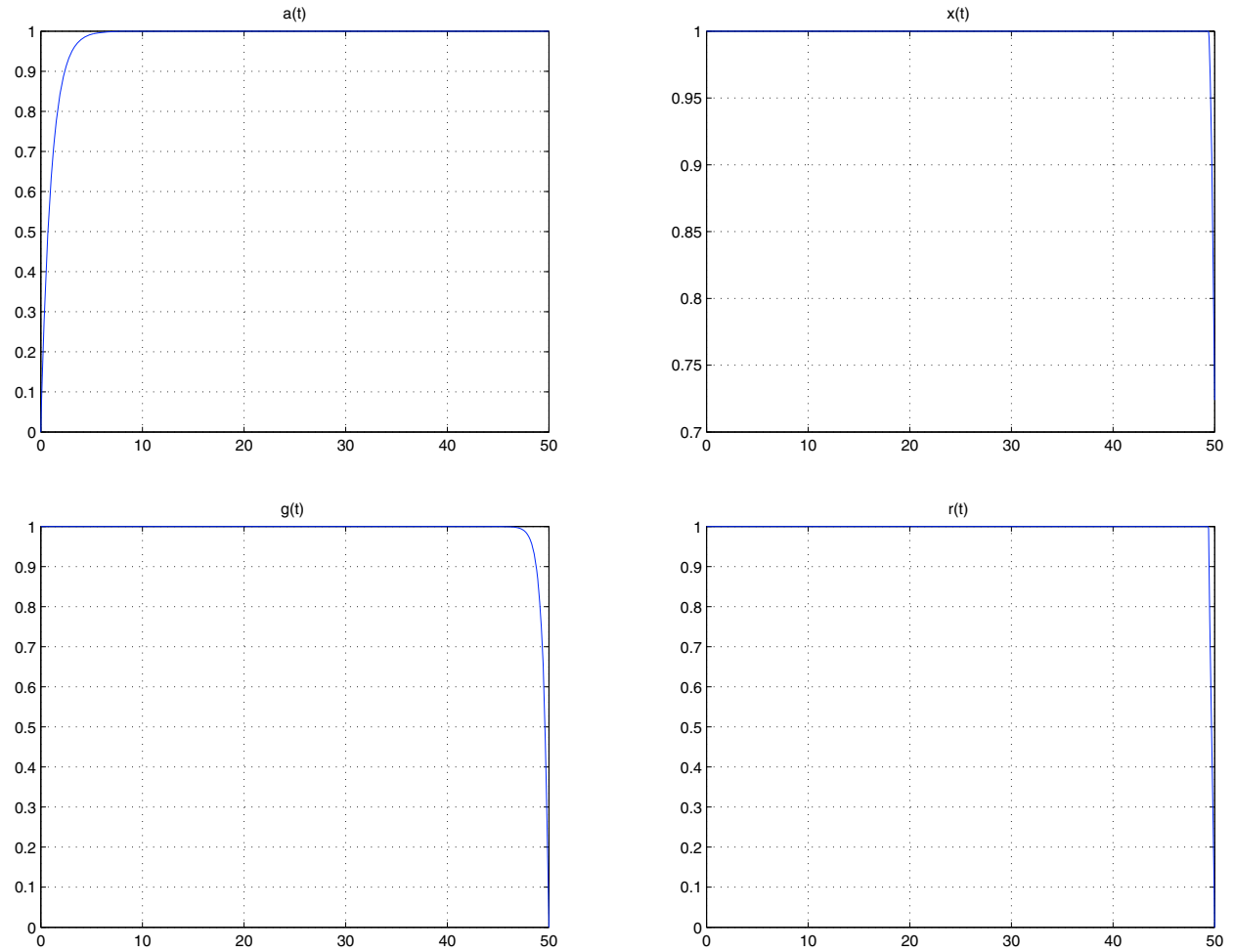

Figure 5. Cost per customer model: numerical solutions for $\gamma=\rho=1, c_{0}=k_{0}=0.5$ and $T=50$. Top left, $a(t)$, top right, $x(t)$, bottom left, $g(t)$ and bottom right, $r(t)$.

The results presented here are potentially important in several ways. They provide detailed information on the solutions of the optimizations problems in time. The formulation and analysis provide a basis for understanding the fundamental, non-dimensional, parameters which govern the acquisition and retention optimization process. These results may provide valuable managerial insight.

One of the observations from this study is that in both models there are effectively two strategies. In the first scenario the costs for acquisition and retention are large with respect to the profit, in which case the optimal strategy is to spend only the 'minimum necessary' to recruit customers and almost nothing to maintain them. On the other hand if the costs are low, the best policy is to spend as much as needed to maintain costumers and spend much on recruiting in the early stages. These strategies are valid for most of the business life. In a retrospective, this result is intuitive.

A non-intuitive result is that in the cost per customer model, in the beginning of the business life customers should be retained at all costs, even if the cost is much larger then the profit. The period in which customers should be kept at all costs depends on the parameters.

It should be noted that these models should be verified in real life scenarios and the cost functions are rarely known. However, as noted above, the models can provide managers an important insight.

The numerical method developed for solving these ODEs can be used in wide verity of applications, such that optimal control, in physics, materials science and more.

The treatment of the optimization problem makes it possible to generalize the results for other optimal acquisition and retention problems. Detailed analysis of other models, such as other constraints on budgets and non monopoly models will be presented elsewhere. 
Acknowledgements. The author would like to thank Barak Libai and Eitan Muller from the Recanati Graduate School of Business Administration at Tel Aviv University for presenting him this problem and for the useful discussions. The author would also like to thank David Levin from the School of Mathematical Sciences at Tel Aviv University for his help.

\section{REFERENCES}

[1] T. Ambler, Abandon lifetime value theories and take care of customers now. Marketing 12 (2001) 18.

[2] C.M. Bender and S.A. Orszag, Advanced mathematical methods for scientists and engineers, McGraw-Hill International Series in Pure and Applied Mathematics. McGraw-Hill, New York (1978).

[3] R.C. Blattberg and J. Deighton, Managing marketing by the customer equity test. Harvard Business Rev. 74 (1996) $136-144$.

[4] R.C. Blattberg, G. Getz and J.S. Thomas, Customer Equity: Building and Managing Relationships as Valuable Assets. Harvard Business School Press, Boston, MA, USA (2001).

[5] H.-C. Chang, D. Gottlieb, M. Marion and B.W. Sheldon, Mathematical analysis and optimization of infiltration processes. J. Scientific Computing 13 (1998) 303-321.

[6] A. Ditkowski, Numerical method for solving non-contiguous initial/final-value problems. J. Scientific Computing (to appear).

[7] A. Ditkowski, D. Gottlieb and B.W. Sheldon, On the mathematical analysis and optimization of chemical vapor infiltration in materials science. ESAIM: M2AN 34 (2000) 337-351.

[8] A. Ditkowski, B. Libai and E. Muller, Optimizing acquisition and retention over time. Marketing Lett. (submitted).

[9] C. Fornell and B. Wernerfelt, Defensive marketing strategy by customer complaint management: A theoretical analysis. J. Marketing Research 24 (1987) 337-346.

[10] S. Gupta and D.R. Lehmann, Customers as assets. J. Interactive Marketing 17 (2003) 9-24.

[11] S. Gupta, D.R. Lehmann and J.A. Stuart, Valuing customers. J. Marketing Research 41 (2004) 7-18.

[12] D. Hanssens, Allocating marketing communication expenditures: A long run view, in Measuring and Allocating Marcom Budgets: Seven Expert Points of View, Marketing Science Institute, Cambridge, MA, USA (2003).

[13] J.E. Hogan, K.N. Lemon and R.T. Rust, Customer equity management: Charting new directions for the future of marketing. J. Service Research 5 (2002) 4-12.

[14] D. Jain and S.S. Singh, Customer lifetime value research in marketing: A review and future directions. J. Interactive Marketing 16 (2002) 34-46.

[15] M. Kamien and N.L. Schwartz, Dynamic Optimization. Second edition, New York: North Holland (1991).

[16] L.Y. Lester, CRM meets Wall Street. Target Marketing 26 (2003) 50-54.

[17] R.J. LeVeque, Numerical Methods for Conservation Laws, Lectures in Mathematics ETH Zürich. Second edition, Birkhäuser Verlag (1992).

[18] W.H. Press, S.A. Teukolsky, W.T. Vetterling and B.P. Flannery, Numerical Recipes in C, The Art of Scientific Computing. Second edition, Cambridge University Press (1992).

[19] W.J. Reinartz and V. Kumar, On the profitability of long-life customers in a non-contractual setting: An empirical investigation and implications for marketing. J. Marketing 64 (2000) 17-35.

[20] W.J. Reinartz, J. Thomas and V. Kumar, Balancing acquisition and retention resources to maximize customer profitability. J. Marketing 69 (2005) 63-79.

[21] R.T. Rust, K.N. Lemon and V.A. Zeithaml, Return on marketing: Using customer equity to focus marketing strategy. J. Marketing 68 (2004) 109-127.

[22] L. Selden and G. Colvin, How to measure the profitability of your customers. Harvard Business Rev. (2003) 74-81.

[23] J.S. Thomas, A methodology for linking customer acquisition to customer retention. J. Marketing Research 38 (2001) $262-268$.

[24] B. Vatanasombut, A.C. Stylianou and M. Igbaria, How to retain online customers. Communication of the ACM 47 (2004) $65-78$.

[25] R. Venkatesan and V. Kumar, A customer lifetime value framework for customer selection and optimal resource allocation strategy. J. Marketing 68 (2004) 106-125.

[26] R. Weinstock, Calculus of Variations. Dover Punlications, Inc., NY (1974).

[27] V.A. Zeithaml, Service quality, profitability, and the economic worth of customers: What we know and what we need to learn. J. Acad. Mark. Sci. 28 (2000) 67-85. 